\title{
SOME THEOREMS ON HOPFICITY
}

BY

\section{R. HIRSHON}

1. Introduction. Let $G$ be a group and let Aut $G$ be the group of automorphisms of $G$ and let End on $G$ be the semigroup of endomorphisms of $G$ onto $G$. A group $G$ is called hopfian if End on $G=$ Aut $G$, that is, a group $G$ is hopfian if every endomorphism of $G$ onto itself is an automorphism. To put this in another way, $G$ is hopfian if $G$ is not isomorphic to a proper factor group of itself.

The question whether or not a group is hopfian was first studied by Hopf, who using topological methods, showed that the fundamental groups of closed twodimensional orientable surfaces are hopfian [5].

Several problems concerning hopfian groups are still open. For instance, it is not known whether or not a group $H$ must be hopfian if $H \subset G, G$ abelian and hopfian and $G / H$ finitely generated. Also it is not known whether or not $G$ must be hopfian, if $G$ is abelian, $H \subset G, H$ hopfian, and $G / H$ finitely generated [2]. On the other hand, A. L. S. Corner [3], has shown the surprising result, that the direct product $A \times A$ of an abelian hopfian group $A$ with itself need not be hopfian.

Corner's result leads us to inquire: What conditions on the hopfian groups $A$ and $B$ will guarantee that $A \times B$ is hopfian? We shall prove, for example, in $\S 3$, that the direct product of a hopfian group and a finite abelian group is hopfian. Also we shall prove that the direct product of a hopfian abelian group and a group which obeys the ascending chain condition for normal subgroups (for short, an A.C.C. group) is hopfian (Theorems 3 and 5 respectively).

In $\S 4$ we examine various conditions on a hopfian group $A$ which guarantee $A \times B$ is hopfian for groups $B$ with a principal series. For example if the center of $A, Z(A)$, is trivial or if $A$ satisfies the descending chain condition for normal groups, (for short, $A$ is a D.C.C. group) then $A \times B$ is hopfian.

Theorem 3 is equivalent to: The direct product of a hopfian group and a cyclic group of prime power order is hopfian. In seeking to generalize this result we note that the normal subgroups of a cyclic group $C_{p^{n+1}}$ of prime power order $p^{n+1}$ form a chain and $C_{p^{n+1}}$ has exactly $n$-proper normal subgroups. We define an $n$-normal group as a group $G$ with exactly $n$-proper normal subgroups such that the normal subgroups of $G$ form a chain. Hence the simplest example of an $n$ normal group is $C_{p^{n+1}}$. (We only consider $n$ finite.) We then consider in $\$ 5$ the direct product $G=A \times B$ of a hopfian group $A$ with an $n$-normal group $B$. In Theorem 16, we show that if $G$ is not hopfian, several anomalies arise with respect to $A$. For instance if $G$ is not hopfian we will show that there are infinitely many

Received by the editors November 6, 1968 . 
homomorphisms of $A$ onto $B$. We show that if $B$ is 0 -normal or 1-normal, $A \times B$ is hopfian.

In $\$ 6$ we explore briefly the concept of super-hopficity. If all homomorphic images of $A$ are hopfian, we say that $A$ is super-hopfian. We show for example that if $G$ is generated by a super-hopfian normal subgroup $A$ and a normal subgroup $B$ such that $B$ has finitely many normal subgroups, then $G$ is super-hopfian.

Unless otherwise stated, $A$ will always designate a hopfian normal subgroup of $G$ and $T$ will designate an element of End on $G$. If $g \in G, O(g)$ will designate the order of $g,|G|$ will designate the cardinality of $G$. If $H \subset G$ and $j$ is a positive integer, $H T^{-j}$ will designate the complete pre-image of $H$ under $T^{j}$.

Finally, the author expresses his appreciation to Professor Donald Solitar for his suggestion to pursue the study of hopficity, for his construction of n-normal groups and for his valuable suggestions and comments given in the formative stages of this paper.

2. Some general theorems. We begin with a result that shows us that in some cases it suffices to consider infinitely generated hopfian groups $A$.

THEOREM 1. If $G$ is a group containing a hopfian subgroup $N$ of index $[G: N]=r$, $r$ finite, such that $G$ contains only finitely many subgroups of index $r$, then $G$ is hopfian.

Proof. Suppose $G \sim G / K, K \neq 1$. If under an isomorphism of $G$ onto $G / K, K$ corresponds to $K_{1} / K$, we see $G \sim G / K \sim G / K_{1}$. Repeating the procedure, we see there exists subgroups $K_{\mathfrak{i}}$, where $K_{\mathfrak{i}}$ is a proper subgroup of $K_{i+1}$ such that

$$
G \sim G / K_{i}, \quad i \geqq 0, K_{0}=K .
$$

Hence we may write $N \sim M_{i} / K_{i}$ so that $[G: N]=\left[G: M_{i}\right]=r$. Hence $M_{i}=M_{j}$ for some $i$ and $j$ with $i<j$. But then,

$$
\frac{M_{i} / K_{i}}{K_{j} / K_{i}} \sim \frac{M_{i}}{K_{j}}=\frac{M_{j}}{K_{j}} \sim N \sim \frac{M_{i}}{K_{i}}
$$

so that $N$ is not hopfian.

The following corollaries follow quite easily:

COROLlaRY 1. Let $G$ be a group containing a hopfian normal subgroup $N$ of index $[G: N]=r$ ( $r$ not necessarily finite) such that $G$ contains only finitely many normal subgroups of index $r$, then $G$ is hopfian.

COROLlaRY 2. If $G$ is a finitely generated group containing a subgroup $N$ of finite index, $N$ hopfian, then $G$ is hopfian.

COROLlaRY 3. If $A$ is finitely generated, and $|B|<\infty$, then $A \times B$ is hopfian.

LEMMA 1. If $G / A$ is hopfian and if $A T \subset A$, then $T \in$ Aut $G$. 
Proof. $T$ induces an endomorphism of $G / A$ onto itself in the obvious way. Since $G / A$ is hopfian we conclude $A T^{-1}=A$ from which the conclusion easily follows.

THEOREM 2. Let $A$ and $G / A$ be hopfian and suppose one of the following holds:

(a) $A \subset Z(G), G / A$ centerless,

(b) A a periodic group, $G / A$ torsion free,

(c) $A$ and $G / A=B$ both periodic groups such that if $a \in A, b \in B$, then $(O(a)$, $O(b))=1$.

Then $G$ is hopfian.

Proof. Apply the previous lemma.

3. $G / A$ an A.C.C. group.

THeOREM 3. If $B$ is a finite abelian group then $G=A \times B$ is hopfian.

Proof. It suffices to assume that $B$ is cyclic of prime power order, say, $|B|=p^{n}$ $B=\langle b\rangle$. Throughout this discussion and the next one, we will use symbols $a, a_{i}$ to designate elements of $A$.

Suppose first for a given $T$, we have $b T=a$. Let $b^{r} a_{1}$ be a pre-image of $b$ under $T$. Let $u=b a_{1}$ and let $v=b a^{-r+1}$. We may then verify,

$$
G=\langle b\rangle \times A=\langle u\rangle A=\langle v\rangle \times A
$$

and $u T=v$. Let $A^{1}=\langle v\rangle T^{-1} \cap A$ so that $\langle v\rangle T^{-1}=\langle u\rangle A^{1}$. Hence,

$$
A \sim(G /\langle v\rangle) \sim\left(G /\langle v\rangle T^{-1}\right)=(\langle u\rangle A) /\left(\langle u\rangle A^{1}\right) \sim A / A^{1} .
$$

Hence $A^{1}=1$. Hence $T$ is an isomorphism on $A$ and without too much difficulty, one sees that $T \in$ Aut $G$.

Now suppose $b T \notin A$, say $b T=b^{q} a$. If $(q, p)=1$, we can find an automorphism $S$ of $G$ such that $b T S=b$, so that by Lemma $1, T S \in$ Aut $G$ and a fortiori, $T \in$ Aut $G$. Hence we may assume $(q, p) \neq 1$.

If $a T \in A$ and if $f_{p}$ designates the greatest power of $p$ dividing the integer $f$ then $b T^{2}=b^{r} a_{2}$ where $r_{p}>q_{p}$. If $a T=b^{s} a_{3}$, and $a T \notin A$, and if $s_{p} \leqq q_{p}$, then for a suitable integer $u$, if $z=b a^{u}, z T \in A$ and $G=\langle z\rangle \times A$. If $s_{p}>q_{p}$ then $b T^{2}=b^{v} a_{4}$ where $v_{p}>q_{p}$. Hence if $(q, p) \neq 1$, we see that we may find an element $w$ of $G$, such that $G=\langle w\rangle \times A$ and $w T^{i} \in A$ for some integer $i, 1 \leqq i<2^{n}$. Hence $T^{i}$ and $T$ are automorphisms.

THEOREM 4. If $A$ is abelian and $B$ is finitely generated and abelian then $G=A \times B$ is hopfian.

Proof. By the previous theorem, we may assume $B=\langle b\rangle \sim C_{\infty}$.

By Lemma 1, if $A \subset A T^{-1}$ then $T \in$ Aut $G$. Hence we may assume $A \mid\left(A \cap A T^{-1}\right)$ is infinite cyclic, that is,

$$
A=\langle a\rangle \times A \cap A T^{-1}
$$


But $A /(A \cap A T)$ is contained isomorphically in $G / A T$ which in turn is a homomorphic image of $G / A$. Hence we may write, $A=\left\langle a_{1}\right\rangle A \cap A T$. Hence there is an element $S, S \in$ End on $A$ which agrees with $T$ on $A \cap A T^{-1}$ such that $a S=a_{1}$. It easily follows that $T \in$ Aut $G$.

COROLlARY. If $A$ is abelian and if $B$ is finitely generated and $B^{\prime}$ the commutator group of $B$ is hopfian then $A \times B=G$ is hopfian.

Proof. $B^{\prime} T=B^{\prime}$ so that $B^{\prime} T^{-1}=B^{\prime}$ or else $(A \times B) / B^{\prime} \sim A \times\left(B / B^{\prime}\right)$ is not hopfian.

Corollary. If $Z(A)$ and $A / Z(A)$ are hopfian and if $B$ is a finitely generated abelian group, then $A \times B$ is hopfian.

Proof. $[Z(A) \times B] T^{-1}=Z(A) \times B$. Now apply the theorem.

We present here some general observations concerning $T$ in relation to $G / A$, where $G$ and $T$ are arbitrary and $G / A$ is an A.C.C. group. ( $A$ need not be hopfian in this discussion.)

We note $T$ induces in a natural way, a homomorphism of $G / A T^{i}$ onto $G / A T^{i+1}$. Since $G / A$ is an A.C.C. group we see that ultimately all these homomorphisms are isomorphisms that is, for $s \geqq r$

$$
\left(A T^{s+j}\right) T^{-j}=A T^{s}, \quad j \geqq 1
$$

so that kernel $T^{j} \subset A T^{s}$. Hence

$$
\text { kernel } T^{j} \subset \bigcap_{s \geqq r} A T^{s}, \quad j \geqq 1 .
$$

It follows that a necessary and sufficient condition that $T \in$ Aut $G$ is that $T^{i}$ be an isomorphism on $A$ for all $i \geqq 1$. Moreover in seeking to prove that $T \in$ Aut $G$ it is not restrictive to assume that, for $i \geqq 1$ and $j \geqq 1$,

$$
G / A T^{i} \sim G / A T^{i+1}, \quad\left(A T^{i+j}\right) T^{-j}=A T^{i}, \quad \text { kernel } T^{j} \subset A T^{i} .
$$

For if $T$ does not obey the above conditions some power $T_{1}$ of $T$ does and we could work with $T_{1}$ instead of $T$. We will assume (1) whenever it is convenient.

We now resume our convention that $A$ is hopfian.

THEOREM 5. If every proper homomorphic image of $A$ is abelian and $B$ is an A.C.C. group then $G=A \times B$ is hopfian.

Proof. Deny. Then we may find $T, T$ not an isomorphism on $A$ such that the conditions (1) hold. Let,

$$
G_{1}=\operatorname{gp}\left(A, A T, A T^{2}, A T^{3}, \ldots\right) .
$$

Then $G_{1} T \subset G_{1}$, so that $G_{1} T^{-1}=G_{1}$. However $A T^{i} \subset Z(G), i \geqq 1$ because $G=A T^{i}$ - $B T^{i}$ and $A T^{i}$ is abelian. Hence $G_{1}=A \times B_{1}$ where $B_{1} \subset Z(B)$. Hence $B_{1}$ is finitely generated so that $A \times B_{1}$ is hopfian which implies $T$ is an isomorphism on $G_{1}$, a contradiction of our hypothesis. 
Corollary. If $A$ is abelian and $B$ is an A.C.C. group, then $A \times B$ is hopfian.

In view of the last theorem, it might be of some interest to give an example of a hopfian group $A$, which is not an A.C.C. group and which is not abelian, but yet every proper homomorphic image of $A$ is abelian. We proceed to do this.

Definition. Let $H$ be a group and $F$ a group of automorphisms. We will say $G$ is an extension of $H$ by $F$, if $G$ consists of elements $f h, f \in F, h \in H$, where multiplication in $G$ is defined by

$$
\left(f_{1} h_{1}\right)\left(f_{2} h_{2}\right)=\left(f_{1} f_{2}\right)\left(h_{1}^{f_{2}} h_{2}\right)
$$

for $f_{i} \in F$ and $h_{i} \in H$, where $h_{1}^{f}$ is the image of $h_{1}$ under $f_{2}$.

THEOREM 6. Let $H$ be a simple group and let $L$ be a hopfian group of automorphism of $H$. Furthermore, suppose

(2) $L \cap$ inner-automorphism $H=1$.

Then if $G$ is an extension of $H$ by $L$ then $G$ is hopfian. In fact if $L$ is super-hopfian, then $G$ is super-hopfian.

Proof. If $N \triangle G$ and $N \neq 1$ then $H \subset N$, for if $H \cap N=1$ the elements of $H$ and $N$ commute element-wise, which leads to a contradiction of (2). Hence, if $T \in$ End on $G$, by Lemma $1, H T \neq 1$. Hence $H \subset H T$. But $H T \sim H$ since $H$ is simple. Hence $H=H T$. By Lemma 1 again, $T \in$ Aut $G$. If $L$ is super-hopfian, every proper homomorphic image of $G$ is a homomorphic image of $L$ so that $G$ is super-hopfian.

As an application, let $H$ be the alternating group on an infinite countable set. Let $p_{i}, i=1,2,3, \ldots$, be a sequence of distinct primes. Then $H$ has a group of automorphisms $L$ which is the restricted direct product of cyclic groups of order $p_{i}, i=1,2, \ldots$, and such that (2) holds. $L$ is super-hopfian. Hence $G$ is not an A.C.C. group, every proper homomorphic image of $G$ is abelian and $G$ is superhopfian.

Somewhat along the lines of the previous theorem, we have

THEOREM 7. Let every proper normal subgroup of $A$ be an A.C.C. group. Let every normal subgroup of $B$ be an A.C.C. group. Then if $G / A \sim B$, then $G$ is hopfian.

Proof. Deny. Suppose $T$ is not an isomorphism on $A$ and kernel $T \subset A T$. Hence

$$
B_{1}=(A \cdot A T) / A \sim A T / A \cap A T \sim A / A_{1} .
$$

Now $B_{1}$ is contained isomorphically in $B$ as a normal subgroup. Hence $A / A_{1}$ is an A.C.C. group. But $A_{1} \neq A$ or else $A T \subset A$ contradicting Lemma 1 . Hence $A_{1}$ is an A.C.C. group. But then so is $A$ and certainly then so is $G$ implying that $G$ is hopfian after all.

We now present some observations concerning the group $G$ where $G / A$ has finitely many normal subgroups. 
Suppose $G$ is not hopfian. Then we may choose $T$ satisfying the conditions (1), $T$ not an isomorphism on $A$. Moreover, we may choose positive integer $r$ and $k$, $r<k$ such that

$$
\begin{gathered}
A \cdot A T^{-k}=A \cdot A T^{-r}=L, \\
A T^{k} \cdot A=A T^{r} \cdot A=M .
\end{gathered}
$$

Hence, $M T^{r-k}=M$, so that $M$ is.not hopfian. If $G / A$ is finite, but $G$ is not hopfian, we might begin by choosing [ $G: A$ ] as small as possible so that if $M$ is constructed as above, $M=G$. But then $G / A$ is a homomorphic image of $A$. We may summarize part of the previous remarks as

THEOREM 8. The statement,

If $A$ is hopfian and $G / A$ is finite then $G$ is hopfian is universally true if and only if the statement,

If $A$ is hopfian and $G / A$ is a finite homomorphic image of $A$, then $G$ is hopfian, is universally true.

4. $A \times B$, where $B$ has a principal series.

Definition. We say that a group $B$ may be cancelled in direct products if whenever

$$
C \times B \sim C^{1} \times B^{1} \text { and } B \sim B^{1}
$$

then $C \sim C^{1}$ (for any $C$ ).

LEMMA 2. If $B$ has a principal series, $B$ may be cancelled in direct products.

Proof. See [4].

THEOREM 9. If $B$ has a principal series, a necessary and sufficient condition for $A \times B$ to be hopfian is that $A T \cap B T=1$ for arbitrary $T$ of End on $(A \times B)$.

Proof. The necessity part of the theorem is clear. Now suppose that $A T \cap B T=1$ for any $T \in$ End on $(A \times B)$. By the remarks preceding (1), we can choose $r>0$ such that

$$
\text { kernel } T^{j} \subset A T^{s} \text { for } j \geqq 1 \text { and } s \geqq r \text {, }
$$

where $r$ depends on $T$. By hypothesis, $A T^{r} \cap B T^{r}=1$ so,

$$
A \times B=A T^{r} \times B T^{r} .
$$

Hence if $K=\operatorname{kernel} T^{r} \cap B$, then

$$
B / K \sim B T^{r} \text { and } A \times(B / K) \sim\left(A T^{r} / K\right) \times B T^{r} .
$$

Hence by Lemma 2 we see $A \sim A T^{r} / K$. It follows without difficulty that $T^{r}$ and $T$ are automorphisms. 
COROLlARY 1. If $B$ has a principal series, then a sufficient condition for $T$ to be an automorphism, for $T$ in End on $(A \times B)$, is

$$
A T^{i} \cap B T^{i}=1, \quad i \geqq 1 .
$$

Corollary 2. A sufficient condition for $T \in$ Aut $(A \times B)$ is kernel $T^{i} \subset A, i \geqq 1$ (where $B$ has a principal series).

COROLlary 3. If $G$ has a principal series and if $T \in$ End on $(A \times B)$, and if $A T$ $\cap B T=1$, and if kernel $T \cap B \subset A T$ then $T$ is an automorphism.

THEOREM 10. If $B$ has a principal series and if there are only finitely many possible kernels for homomorphisms of $A$ into normal subgroups of $B$, then $A \times B$ is hopfian.

Proof. Choose $T$ obeying the conditions (1). Then write,

$$
A \cdot A T^{k}=A \times B_{k}, \quad k \geqq 1, B_{k} \triangle B .
$$

The above gives rise to a homomorphism of $A$ onto $B_{k}$, whose kernel is $A \cap A T^{-k}$. Hence we have for say $0<r<s$,

$$
A \cap A T^{-r}=A \cap A T^{-s} .
$$

Hence, $A T^{s} \cap A T^{s-r}=A T^{s} \cap A$, so that kernel $T^{i} \subset A, i \geqq 1$ and we may apply Corollary 2, of the previous theorem.

Corollary 1. If $B$ is finite and $A$ has only finitely many normal subgroups $A_{*}$ such that $\left[A: A_{*}\right]$ is a divisor of $[B: 1]$, then $A \times B$ is hopfian.

COROLlaRY 2. If $B$ has a principal series and if there are only finitely many homomorphisms of $A$ into $B$, then $A \times B$ is hopfian.

LEMMA 3. Let $B$ be a group with a principal series. Let $P$ be a property of groups such that:

(a) $A$ has a nontrivial normal group $A_{*}$ such that $A / A_{*}$ has property $P$.

(b) If $A$ has property $P$, then $A \times B$ is hopfian.

(c) If $A^{*} \triangle A$ and $A / A^{*}$ has $P$ and if $T \in$ End on $(A \times B)$, then $A /\left(A^{*} \cap A^{*} T^{j}\right)$ has property $P$ for all integers $j$.

(d) $A$ satisfies the descending chain condition for normal subgroups $A^{*}$ such that $A / A^{*}$ has property $P$.

Then $A \times B$ is hopfian.

Proof. Choose a minimal normal group $A^{*}$ such that $A^{*} \neq 1$ and $A / A^{*}$ has property $P$. Then we may assume $A^{*} T^{j} \cap A^{*}=A^{*}$ for any $j$ so that $A^{*} T^{-j}=A^{*}$ for $j \geqq 0$. Now apply Corollary 2 of Theorem 9 .

THEOREM 11. Suppose either

(a) $B$ is finite, and $A$ satisfies the descending chain condition for normal subgroups of finite index, or 
(b) $B$ has a composition series and A satisfies the descending chain condition for normal subgroups $A^{*}$ such that $A / A^{*}$ has a composition series, or

(c) $B$ has a principal series and $A$ satisfies the descending chain condition for normal groups $A^{*}$ such that $A / A^{*}$ has a principal series.

Then $G=A \times B$ is hopfian.

Proof. For instance, for (c) take $P$ the property of having a principal series. Let $A / A^{*}$ have property $P$. Let

$$
H=A / A_{1}, \quad E=A^{*} / A_{1}, \quad F=A / A^{*}
$$

where $A_{1}=A^{*} T^{j} \cap A^{*}$. One can show $E$ obeys the ascending and descending chain conditions for normal subgroups of $H$, that is any ascending or descending chain of subgroups of $E$ which are normal in $H$ terminates. It follows that $H$ has a principal series.

Corollary. If $A$ is a D.C.C. group, and if $B$ has a principal series, then $A \times B$ is hopfian.

THEOREM 12. If $A$ satisfies the ascending chain condition for normal nonhopfian subgroups, and if $B$ has a principal series, then $G=A \times B$ is hopfian.

Proof. Deny. Choose $T$ satisfying the conditions (1), but $T$ not an isomorphism on $A$. Let,

$$
A_{i}=\bigcap A T^{q \cdot 2^{i}}, \quad i=0,1,2, \ldots
$$

where $q$ ranges over all integers. Then $A_{i} T^{2 i}=A_{i}$ so that the $A_{i}$ are nonhopfian. Hence we may find $j$ so that $A_{j}=A_{j+1}$. Hence $A_{j+1} T^{2^{j}}=A_{j}$. It follows that kernel $T^{i} \subset A, i \geqq 1$. Now apply Corollary 2 of Theorem 9 to obtain a contradiction.

In view of the former result, it might be interesting to give an example of a hopfian group $G$ such that $G$ contains a normal nonhopfian subgroup and such that $G$ obeys the ascending chain condition for normal nonhopfian subgroups. (The example we give will be of special interest in Theorem 18.)

Let $p$ be a prime and $K$ be the field with $p$ elements. Let $m$ be an integer, $m \geqq 3$. Let SL $(m, K)$ be the group of nonsingular, unimodular, linear transformations of a vector space $V$ of dimension $m$ over $K$. Let

$$
\operatorname{PSL}(m, K)=\operatorname{SL}(m, K) \mid Z
$$

where $Z=$ center of $\operatorname{SL}(m, K)$.

LEMMA 4. $Z$ is the subgroup of diagonal linear transformations of $\operatorname{SL}(m, K)$ i.e., $Z$ consists of those transformations $T$ which have the form

$$
x T=\lambda x, \lambda^{m}=1 \text { for all } x \in V .
$$

Also, PSL $(m, K)$ is simple. 
Proof. This is a special case of a more general result. See [6].

Now let $\left\langle a_{i}\right\rangle$ be a cyclic group of order $p, i=1,2,3, \ldots$ Let $G$ be the restricted direct sum of the $\left\langle a_{i}\right\rangle$. Let $G_{r}$ be the direct sum of the groups $\left\langle a_{i}\right\rangle$ for $1 \leqq i \leqq r$ and let $G^{r}$ be the restricted direct sum of the groups $\left\langle a_{i}\right\rangle$ for $i>r$. Hence $G$ is the direct sum of $G_{r}$ and $G^{r}$. Now let $F_{*}$ be the set of automorphisms $T$ of $G$ such that there exists an $r$ such that $T$ fixes the group $G_{r}$, that is $G_{r} T=G_{r}$, and such that $T$ is the identity map on $G^{r}$, i.e., if $x \in G^{r}, x T=x$. One can see that $F_{*}$ is a group of automorphisms of $G$. Now if $T \in F_{*}$ we may choose $r$ such that $G_{r} T=G_{r}$ and $T$ is the identity on $G^{r}$. Now on $G_{r}, T$ acts as a linear transformation and we define $|T|$ as the determinant of the matrix representing $T$ on $G_{r}$. It may be verified that $|T|$ is well defined, and independent of $r$. Now let $F$ be the subgroup of $F_{*}$ of those transformations $T$, with $|T|=1$. We claim that $F$ is simple. To see this let $F_{n}$ be those elements $T$ of $F$ such that $G_{p^{n}} T=G_{p^{n}}$, and $T$ the identity on $G^{p^{n}}$. We see $F$ is the union of the $F_{n}$. Since the union of an ascending sequence of simple groups is simple, we need only show that the groups $F_{n}$ are simple. However one can see that $F_{n} \sim \mathrm{SL}\left(p^{n}, K\right)$ and since $\lambda^{p^{n}}=\lambda$ in $K, \operatorname{SL}\left(p^{n}, K\right)$ has no center and so is simple by the previous lemma.

Now let $M$ be the extension of the group $G$ by $F$. One sees that if $g_{1}$ and $g_{2}$ are elements in $G, g_{1} \neq 1, g_{2} \neq 1$, there exists $T \in F$ such that $g_{1} T=g_{2}$. One can now see that $G$ is the only normal subgroup of $M$ so that certainly $M$ is hopfian and has a nonhopfian normal subgroup, namely $G$, and $M$ obeys the ascending chain condition for normal nonhopfian groups.

LEMMA 5. Let $C \triangle G$ and suppose that $C$ has finitely many normal subgroups. If $T \in$ End on $G$, then either $C \cap C T^{i}=1$ for all positive $i$ sufficiently large, or we can find $C^{*}, C^{*} \subset C, C^{*} \triangle G, C^{*} \neq 1$, and a positive integer $j$ such that $C^{*} T^{j}=C^{*}$.

Proof. If $C \cap C T^{i} \neq 1$, for all $i$ sufficiently large, we may find positive integers $r$ and $s, r<s$, and normal groups $C_{*}$ and $C^{*}$ of $C$ such that if $u$ is either $r$ or $s$,

$$
C T^{u} \cap C=C_{*} T^{u}=C^{*} \neq 1 .
$$

Hence if $j=s-r, C^{*} T^{j}=C^{*}$.

We note at this point that if $A$ is hyper-hopfian, that is if every normal subgroup of $A$ is hopfian, then certainly Theorem 12 guarantees $A \times B$ is hopfian if $B$ has a principal series. For instance if the groups $M_{i}$ are torsion-hyper-hopfian groups such that elements $m_{i}, m_{j}$ of $M_{i}$ and $M_{j}$ respectively, $i \neq j$, have relatively prime orders, then the restricted direct product of the $M_{i}$ is hyper-hopfian. In particular one may choose the $M_{i}$ to be finite groups.

THEOREM 13. If $A \times B$ is not hopfian and $B$ has a principal series, then there exists a homomorphic image $C$ of $B$ such that $A \times C$ is not hopfian, and $Z(C) \neq 1$, and if $T$ is an arbitrary element of End on $(A \times C)$, then $T$ is an isomorphism on $C$. Also if $C_{1} \triangle C, C_{1} \neq 1$, then $A \times\left(C / C_{1}\right)$ is hopfian. Furthermore, if $B$ has finitely many normal subgroups, and $A \times B$ is not hopfian, we can find $C$ with the former 
properties, and in addition with the property that if $T \in$ End on $(A \times C), T \notin$ Aut $(A \times C)$, then $C T^{i} \cap C=1$, for all positive i sufficiently large.

Proof. Choose a group $C, C$ a homomorphic image of $B$, with the number of terms in a principal series for $C$ minimal with respect to $A \times C$ being nonhopfian. This guarantees that for all $T \in$ End on $(A \times C), T$ is an isomorphism on $C$ and $A \times C / C_{1}$ is hopfian if $C_{1} \neq 1$. Furthermore, since $A \times C$ is not hopfian, we may choose $T \in$ End on $(A \times C)$ so that $A T \cap C T \neq 1$. Hence $Z(C T) \sim Z(C) \neq 1$. Furthermore if $B$ has finitely many normal subgroups, so does $C$ so that if $T$ is any element of End on $(A \times C), T \notin$ Aut $(A \times C)$, then $C T^{i} \cap C=1$ for all $i$ sufficiently large or else we could choose $C^{*}$ as in the previous lemma and $A \times\left(C / C^{*}\right)$ would not be hopfian.

COROLlary. Suppose A cannot be written in the form

$$
A=A_{1} \cdot A_{2}, A_{i} \triangle A, A_{i} \neq A, i=1,2,
$$

$A_{1}$ and $A_{2}$ commute elementwise, $A_{1}$ a homomorphic image of $A, Z\left(A_{2}\right) \neq 1$.

Then if $B$ has finitely many normal subgroups, $A \times B$ is hopfian. Moreover, if the homomorphic images of $A$ are indecomposable as a direct product, then $A \times B$ is hopfian. Finally if $B$ is fixed, and $A$ cannot be written in the form (3) with the additional stipulation that $A_{2}$ be a homomorphic image of $B$, then $A \times B$ is hopfian.

Proof. If $A \times B$ is not hopfian, choose $C$ as in the previous theorem and $T \in$ End on $(A \times C), C \cap C T=1$, and $T$ not an isomorphism on $A$. Let $N=C T^{-1}$ so that $(A \times C) / N \sim A$ so that we may take $A_{1}=(A N) / N$, and $A_{2}=(C N) / N \sim C$. If $A$ is written in the form (3), then $A / A_{1} \cap A_{2}=A_{1} / A_{1} \cap A_{2} \times A_{2} / A_{1} \cap A_{2}$.

THEOREM 14. If $Z_{0}=1$ and $Z_{n+1} / Z_{n}=Z\left(A / Z_{n}\right), n \geqq 0$, and $A / Z_{n}$ and its center are hopfian for all $n \geqq 0$, then if $B$ has a principal series, $A \times B$ is hopfian.

Proof. Deny. Choose a group $E$ with a principal series and an integer $r \geqq 0$, such that $H=A / Z_{r} \times E$ is not hopfian and $\triangle(E)=$ length of a principal series for $E$ is minimal. That is, if $A / Z_{q} \times D$ is not hopfian, and if $D$ has a principal series, then $\triangle(E) \leqq \triangle(D)$. Consequently, the group $C$ we may associate with $E$, by the previous theorem, is $E$ itself, so $Z(E) \neq 1$. If $T \in$ End on $H$, but $T \notin$ Aut $H$, we see from the minimality of $\Delta(E)$ that

$$
Z(H) T^{-1}=Z(H)=Z_{r+1} \mid Z_{r} \times Z(E) .
$$

However, $Z(E)$ is finite and this contradicts Theorem 3.

Corollary. If $Z(A)=1$, and if $A$ is hopfian and $B$ has a principal series, then $A \times B$ is hopfian.

5. $A \times B, B$-normal. We begin by giving some examples of $n$-normal groups. As we have mentioned, we have the groups $C_{t}, t=p^{n+1}, p$ a prime. Or if $F$ is a 
simple group and $B$ is an $n$-normal group of automorphisms of $F$, such that $B$ does not contain any inner-automorphism (different from 1), then the extension of $F$ by $B$ is $n+1$ normal. In particular, if $B \sim C_{p^{n}}, p$ a prime, we can find a prime $q$, $q=1 \bmod p^{n}$ so that $C_{q}$ has a group of automorphisms, $B$, and extending $C_{q}$ by $B$ gives us a nonabelian $n$-normal group. Similarly if $H$ is the alternating group of arbitrary infinite cardinality, and if $R \in$ Aut $H, O(R)=p^{n}$, and if $\langle R\rangle$ contains no inner-automorphism except 1 , if we extend $H$ by $\langle R\rangle$ we obtain an infinite $n$-normal group so that nonabelian $n$-normal groups of arbitrary infinite cardinality exist.

Until further notice, $B$ shall represent an $n$-normal group, with normal subgroups,

$$
1=B_{0}, B_{1}, \ldots, B_{n}, B_{n+1}=B, B_{i} \subset B_{i+1} .
$$

Lemma 6. If $T \in$ End on $G, G=A \times B, T \notin$ Aut $G$, then $G=A \cdot A T$ and $B \cdot A T$ is $a$ proper subgroup of $G$.

Proof. Either $A \cdot A T \subset B \cdot A T$ or $B \cdot A T \subset A \cdot A T$ : Hence all we need show is that $A \cdot A T$ is not a subgroup of $B \cdot A T$. But if $A \cdot A T \subset B \cdot A T$, then $G=B \cdot A T$ and hence $A \sim A T \mid B \cap A T$, from which we could easily deduce that $T \in$ Aut $G$.

Lemma 7. Suppose $T \in$ Aut $G$, and $A T \cap B T=B_{i} T$ where kernel $T \cap B=B_{k} \subset B_{i}$. Then if $B \cap A T=B_{j}$, then $j>i$.

Proof. Use the previous lemma to see that $B / B_{j}$ is contained isomorphically as a proper normal subgroup of $G|A T \sim B| B_{i}$.

LEMMA 8. If $B T \sim B$, then $A T \cap B T \neq 1$.

Proof. Deny. Then $G=A \times B=A T \times B T$. By the previous lemma, $B_{k} \subset A T$. But then Corollary 3 of Theorem 9 implies that $T \in$ Aut $G$.

Theorem 15. A necessary and sufficient condition that $T \in$ Aut $G$ is $A T \cap B T=1$.

Proof. The previous lemma and Lemma 2.

In our next theorem, we show that if $A \times B$ is not hopfian $A$ must enjoy several anomalous properties.

THEOREM 16. Suppose $G=A \times B$ is not hopfian. Then,

(1) There exists infinitely many homomorphisms of $A$ onto $B$.

Also there exist normal subgroups of $A \times B, R^{*}, R, R_{\mathfrak{i}}, R^{i}, i \geqq 0$ such that

(2) $R^{*} \subset R^{i+1} \subset R^{i}, R^{0}=R_{0}, R_{i} \subset R_{i+1} \subset R$ for all $i$.

(3) $R^{*}=\bigcap R^{i}, R=\bigcup R_{i}$, where the intersection and union are taken over all

$i \geqq 0$. Also the containments in (2) are proper.

(4) The $R^{i}$ are subgroups of $A$.

(5) $R^{*}$ and $R$ are not hopfian.

(6) $R^{i} / R^{i+1} \sim R^{j} / R^{j+1} \sim R_{1} / R_{0} \sim a$ normal subgroup of $B$ for all $i$ and $j$, and $R^{i} / R^{*} \sim R^{j} / R^{*}$ for all $i$ and $j$.

(7) $R_{i+1} / R_{i} \sim R_{j+1} / R_{j} \sim a$ normal subgroup of a proper homomorphic image of $B$, $i \geqq 1, j \geqq 1$ and 
(8) There exist normal subgroups $A_{i} \subset A, i=1,2, \ldots, A_{i} \subset A_{i+1}$ properly, such that $A_{i+1} / A_{i} \sim R_{2} / R_{1}$ for all $i$.

(9) There exist normal subgroups $K_{i}, K_{i} \subset K_{i+1}, i \geqq 0$, such that if $L=\bigcup K_{i}$, then $L$ is nonhopfian, and

$$
R_{i} / K_{j} \sim R_{i+j}, \quad R_{i} / L \sim R_{j} / L, \quad K_{i+j} / K_{j} \sim K_{i} \quad \text { and } L / K_{j} \sim L .
$$

Proof. Let $T \in$ End on $G, T \in$ Aut $G$. Then by Lemma $6, A \cdot A T^{j}=G$ for all $j>0$, which implies $A \cdot A T^{-j}=G$ for $j>0$. Hence $A / A \cap A T^{-j} \sim B$ and one may show (as in Theorem 10) that if the groups $A \cap A T^{-j}, j=1,2,3, \ldots$ are not distinct, then $T$ is an automorphism.

Now let us assume, without loss of generality, that $T$ satisfies the condition (1), and that $A T^{r} \cap B T^{r}=B_{i} T^{r}$ for all $r \geqq 1$ (for some fixed $i, i \geqq 1$ ) and that $i$ is maximal in the sense that if

$$
B_{u} T^{q} \subset A T^{q} \cap B T^{q} \text { for some } q \geqq 1 \text {, then } u \leqq i .
$$

(For if $T$ does not obey these conditions, some power of $T$ does, and we could then work with this power of $T$.)

Now we define,

$$
R_{j}=\bigcap_{i \geqq j} A T^{i} \quad j \geqq 0, R=\bigcup_{j \geqq 0} R_{j} .
$$

With the aid of (1), we see $R_{i} T=R_{i+1}$ so that $R T=R$, and $R \neq 1$, since kernel $T \subset R$. Moreover, the groups $R_{j}$ are all distinct, for if say $R_{m}=R_{m+1}$, then $R_{j}=R_{m}$ for $j>m$ and hence $R=R_{m}$. But then with the aid of Lemma 7, we see $B_{i+1} \subset R_{1} \subset R$. Hence,

$$
A \cdot R=A \times B_{s}, \quad s>i .
$$

Hence, $(A \cdot R) T^{m}=A T^{m} \cdot R T^{m}=A T^{m} R=A T^{m}=A T^{m} \cdot B_{s} T^{m} \quad$ so that $\quad B_{s} T^{m} \subset B T^{m}$ $\cap A T^{m}$, a contradiction of the maximality of $i$.

We now define

$$
R^{0}=R_{0} \quad \text { and } \quad R^{n+1}=R^{n} T^{-1} R_{0}, \quad n \geqq 0 .
$$

By induction and the previous lemma, we see that $R^{n+1}$ is a proper subgroup of $R^{n}$ and $R^{n}=\bigcap A T^{j}$ where $j$ ranges over all integers $\geqq-n$ for each $n \geqq 0$. Moreover if we consider the homomorphism of $R^{n}$ onto $R^{n-1}$, induced by $T$ for $n \geqq 1$, we see that the preimage of $R^{n}$ is exactly $R^{n+1}$ so that

$$
R^{n} / R^{n+1} \sim R^{n-1} / R^{n}, \quad n \geqq 1 .
$$

Furthermore, if we consider the homomorphism of $R^{0}=R_{0}$ onto $R_{1}$ induced by $T$, we see that the preimage of $R_{0}$ is exactly $R^{1}$ so that $R^{0} / R^{1} \sim R_{1} / R_{0}$.

Now one may see that $R_{1} / R_{0}$ is isomorphic to a normal subgroup of $A T / A$ $\cap A T \sim B$. Also with the aid of (1) we see,

$$
R_{j+1} / R_{j} \sim R_{j+2} / R_{j+1}, \quad j>1 .
$$

Furthermore, $R_{2} / R_{1}$ is isomorphic to a normal subgroup of $A T^{2} / A T \cap A T^{2} \sim B / B_{i}$. 
If $A_{k}=R_{k} \cap A$, ultimately the $A_{k}$ are distinct and by a suitable reindexing, the $A_{k}$ may be seen to have the properties asserted in the Theorem. One may verify the remaining assertions by taking $K_{j}=\operatorname{kernel} T^{j}, j \geqq 1$, and $L=\bigcup_{j \geqq 1} K_{j}$, and by noting that $R^{*} T^{-1}=R^{*}$.

Corollary. If $|B|>|A|$, then $A \times B$ is hopfian.

Proof. $B$ cannot be a homorphic image of $A$.

We now find some particular values of $n$ for which $A \times B$ is hopfian.

LEMMA 9. If $|B|=p^{n+1}, p$ a prime, then $B \sim C_{p}^{n+1}$.

Proof. Use induction on $n$, and the fact that $Z(B) \neq 1$.

LEMMA 10. If $T \in$ End on $(A \times B)$ and $B T \subset A$ and $B \subset A T$, then $T$ is an isomorphism on $A$.

Proof. $A T=B \times A \cap A T$ and $A=B T(A \cap A T)$. These two decompositions give rise to a homomorphism $S$ of $A T$ onto $A$ such that $S$ agrees with $T$ on $B$ and $S$ is the identity on $A \cap A T$.

LEMMA 11. Let $k$ be the least integer, $k \geqq 0$ (if one exists), such that $A \times B$ is not hopfian for some $A$ and for some $k$ normal group $B$. Then if $T \in$ End on $(A \times B)$, $T \notin$ Aut $(A \times B)$, then $B \cap B T=1$ and $T$ is an isomorphism on $B$.

Proof. Deny. Then $B_{1} T \subset B_{1}$ and $A \times B / B_{1}$ is not hopfian, which contradicts the minimality of $k$ if $B_{1} \neq B$, or the hopficity of $A$ if $B_{1}=B$.

THEOREM 17. If $B$ is $n$-normal, $0 \leqq n \leqq 1$, then $A \times B$ is hopfian.

Proof. Let $k$ be as in the last lemma, $A \times B$ not hopfian, $B k$-normal. We will show $k \geqq 2$. Let $T \in$ End on $(A \times B), T$ not an isomorphism on $A$. Let $A \cdot B T=A \times B_{r}$, $B \cdot A T=\left(B_{q} T\right)(A T), A T \cap B T=B_{i} T, B \cap A T=B_{j}$ where $1 \leqq i<j$. Using Lemma 11 we see $B_{r}, B_{q}$ and $B_{i}$ are central groups of $B$ and hence are cyclic $p$ groups for some prime $p$. Furthermore, we see $A \cap B T=\left(B_{k-r+1}\right) T$ and $B / B_{j} \sim B_{q} / B_{i}, B / B_{k-r+1} \sim B_{r}$, $q=k+i-j+1$. Hence we must have,

$$
j>r, \quad j>q, \quad k-r+1>q, \quad k-r+1>r
$$

or otherwise $B$ would be a finite $p$ group and hence $B$ would be cyclic, a contradiction of Theorem 3. In summary we have,

$$
0 \leqq r<\frac{k+1}{2} \leqq \frac{k+i}{2}<\frac{k+i+1}{2}<j \leqq k+1 .
$$

And with the aid of Lemma 10 , we see $1 \leqq i<j-r \leqq k$. Hence we see $k=0$ or 1 is impossible.

Corollary 1. If $C=D \cdot E, D \triangle C, D \cap E=1$ where $D$ and $E$ are simple, then $A \times C$ is hopfian. 
Proof. Either $C$ is 1-normal or $C \sim D \times E$.

CoRollary 2. If $B$ is 2-normal and if $T \in$ End on $(A \times B)$, $T$ not an automorphism, then $B \subset A T, A \cap B T=B_{2} T, B / B_{2} \sim B_{1} \sim C_{p}$ for some prime $p$ and $B_{1}=Z(B)$.

COROllary 3. If $r$ is a positive integer, then $A \times$ symmetric $(r)$ is hopfian.

Proof. Symmetric 4 is 2 -normal and centerless. If $r \neq 4$, symmetric $r$ is 1 -normal.

COROllary 4. If $B$ is a group such that $B$ has exactly one normal group in a principal series, i.e., $B$ has a principal series of the form $1, B_{*}, B$, then $A \times B$ is hopfian.

Proof. Either $B$ is 1-normal or $B$ is the direct product of simple groups.

Corollary 5. If $G=A \times B, B$-normal and if $B T$ is $i$-normal, $i=0$ or 1 , then $T \in$ Aut $G$.

THEOREM 18. Let $E$ be a class of hopfian groups such that any hopfian group is isomorphic to a unique group of $E$. Then there exists a class $E_{*}$ of hopfian groups such that:

(a) $E$ and $E_{*}$ have the same cardinality.

(b) No two distinct groups of $E_{*}$ are isomorphic.

(c) Any hopfian group is contained isomorphically as a normal subgroup of some group in $E_{*}$.

(d) Every group in $E_{*}$ has a nonhopfian normal subgroup.

Proof. Let $E_{*}$ be the set of groups which is formed by taking the direct product of groups in $E$ with the group $M$ of the example following Theorem 12, i.e. $E_{*}=\{(A \times M) / A \in E\}$.

Our assertions follow from the previous theorem, the definition of $M$ and Lemma 2.

6. Super-hopficity. We terminate this paper with an investigation of the concept of super-hopficity. For an illustration of super-hopficity, we note that the restricted direct product of periodic super-hopfian groups $M_{i}$, such that $\left(O\left(m_{i}\right), O\left(m_{j}\right)\right)=1$ for $m_{i} \in M_{i}, m_{j} \in M_{j}, i \neq j$, is super-hopfian. In particular, the $M_{i}$ might be chosen as finite groups.

We no longer assume that $B$ designates an $n$-normal group.

Lemma 12. Let $A$ be super-hopfian and let $H=A \cdot B, A \triangle H, B \triangle H$. Suppose $T \in$ End on $H$ and $B \subset R, R \triangle H$ and $R T \subset R$. Then $R T^{-1}=R$.

Proof. If $R T^{-1} \neq R, H / R T^{-1}$ is a homomorphic image of $A$, but $H / R T^{-1}$ is not hopfian.

COROLlaRY. If $H$ and $T$ are as in the lemma, and if $r>0$ and if $L_{r}$ is the subgroup of $H$ generated by the groups $B T^{i r}, i \geqq 0$, then $L_{r} T^{-r}=L_{r}$. 
LEMMA 13. If $H$ and $L_{r}$ and $T$ are as in the preceding corollary and if $B \cap B T^{i r}=1$ for fixed $r$ and for all $i \geqq 1$, then $B$ abelian.

Proof. Since $L_{r} T^{r}=L_{r}, L_{r}$ is generated by the groups $B T^{i r}, i \geqq 1$, and $B$ commutes element-wise with each $B T^{i r}, i \geqq 1$. Hence $B \subset Z\left(L_{r}\right)$.

TheOREM 19. Let $H=A \cdot B$ where $A \triangle H$ and $B \triangle H$ and where $A$ is super-hopfian. Suppose $B$ satisfies any one of the following conditions:

(a) $B$ is a finitely generated A.C.C. group.

(b) $B$ has finitely many normal subgroups or,

(c) $B$ is an A.C.C. group and if $B_{*}$ is any homomorphic image of $B$ and if $B_{1} \triangle B_{*}$ and $B_{2} \triangle B_{*}$ and if $B_{1} \sim B_{2}$ then $B_{1}=B_{2}$.

Then $H$ is super-hopfian.

Proof. It suffices to prove $H$ is hopfian since any homomorphic image of $H$ satisfies the same hypothesis as $H$ in any of the three situations. Let us assume that (a) holds. Let $T \in$ End on $H$. In the notation of the corollary to Lemma 12, we have $B \subset L_{1}=L_{1} T$ and $L_{1} T$ is generated by the groups $B T^{i}, i \geqq 1$. Hence, since $B$ is finitely generated, we can find $r$ such that

$$
B \subset B T \cdot B T^{2} \cdots B T^{r-1} B T^{r}=E .
$$

Hence,

$$
B T \subset B T^{2} \cdot B T^{3} \cdots B T^{r} \cdot B T^{r+1}=E T .
$$

Consequently, $E \subset E T$ and hence,

$$
E T^{i} \subset E T^{i+1}, \quad i \geqq 0 .
$$

Now since $B$ is an A.C.C. group, so is $B T^{i}, i \geqq 0$, and hence so is $E$. Consequently, $T$ is an isomorphism on $E T^{i}$ for all $i$ sufficiently large and positive. However, $L_{1}$ is the union of the groups $E T^{i}, i \geqq 1$. Hence in view of (4), we see $T$ is an isomorphism on $L_{1}$. But from the corollary to Lemma $12, L_{1}=L_{1} T^{-1}$ and so $T$ is an automorphism.

Now suppose the assertion of (b) is false and choose a counterexample $A \cdot B=H$ so that $B$ has the fewest number of normal subgroups among all possible counterexamples. Let $T \in$ End on $H, T$ not an isomorphism on $A$. Then we can find $r>0$ such that $B \cap B T^{i}=1$ for all $i \geqq r$ or else by Lemma 5 , we can find $j>0$ such that $B_{*} T^{j}=B_{*}$ for some normal subgroup, $B_{*}$ of $B, B_{*} \neq 1$. Furthermore, $T^{j}$ is an isomorphism on $B$ because of the "minimality" of $B$. Hence,

$$
H / B_{*}=\left[\left(A B_{*}\right) /\left(B_{*}\right)\right]\left(B / B_{*}\right)
$$

is not hopfian, which contradicts the "minimality" of $B$. Hence $r$ exists as asserted, and so we see from Lemma 13 that $B$ is abelian. Hence $B$ is finite. This contradicts part (a) of our theorem.

Finally for (c) we may proceed by denying that $G$ is hopfian. Hence we may choose $B^{*}$ and $A^{*}$ such that $H^{*}=A^{*} \cdot B^{*}, A^{*} \triangle H^{*}, B^{*} \triangle H^{*}, A^{*}$ super-hopfian, $B^{*}$ a 
homomorphic image of $B, H^{*}$ not hopfian, and such that if $H_{1}=A_{1} \cdot B_{1}, A_{1}$ superhopfian, $B_{1}$ a proper homomorphic image of $B^{*}$, then $H_{1}$ is hopfian.

Choose $T \in$ End on $H^{*}, T$ not an isomorphism on $A^{*}$. Note $T^{i}$ must be an isomorphism on $B^{*}$ for $i \geqq 1$. Now if $B^{*} \cap B^{*} T^{j} \neq 1$ for some $j, j \geqq 1$, we may write $B_{*}=B_{2} T^{j}, B_{*} \subset B^{*}, B_{*} \neq 1, B_{*} \neq B_{2}$ (or else $G / B_{*}$ is not hopfian, etc.) but $B_{*} \sim B_{2}$, a contradiction of our hypothesis. Hence, $B^{*} \cap B^{*} T^{j}=1$ for $j \geqq 1$, so that $B^{*}$ is abelian and finitely generated, a contradiction of part (a) of our theorem.

\section{REFERENCES}

1. G. Baumslag and D. Solitar, Some two generator one relator non-hopfian groups, Bull. Amer. Math. Soc. 68 (1962), 199-201.

2. G. Baumslag, "Hopficity and Abelian groups," in Topics in abelian groups, Scott, Foresman and Co., Chicago, Ill., 1963, pp. 331-335.

3. A. L. S. Corner, Three examples on hopficity in torsion-free abelian groups, Acta Math. 16 (1965), 303-310.

4. R. Hirshon, On cancellation in groups, Amer. Math. Monthly (to appear).

5. W. Magnus, A. Karras and D. Solitar, Combinatorial group theory, Interscience, New York, 1966, p. 415.

6. J. Rotman, The theory of groups, Allyn and Bacon, Boston, Mass., 1965, p. 158.

POLYTECHNIC INSTITUTE OF BROOKLYN,

BROOKLYN, New YORK 